NBER WORKING PAPER SERIES

\title{
MEESE-ROGOFF REDUX: MICRO-BASED EXCHANGE RATE FORECASTING
}

\author{
Martin D.D. Evans \\ Richard K. Lyons \\ Working Paper 11042 \\ http://www.nber.org/papers/w11042 \\ NATIONAL BUREAU OF ECONOMIC RESEARCH \\ 1050 Massachusetts Avenue \\ Cambridge, MA 02138 \\ January 2005
}

The views expressed herein are those of the author(s) and do not necessarily reflect the views of the National Bureau of Economic Research.

(C) 2005 by Martin D.D. Evans and Richard K. Lyons. All rights reserved. Short sections of text, not to exceed two paragraphs, may be quoted without explicit permission provided that full credit, including (C) notice, is given to the source. 
Meese-Rogoff Redux: Micro-Based Exchange Rate Forecasting

Martin D.D. Evans and Richard K. Lyons

NBER Working Paper No. 11042

January 2005

JEL No. F3, F4, G1

\section{ABSTRACT}

This paper compares the true, ex-ante forecasting performance of a micro-based model against both a standard macro model and a random walk. In contrast to existing literature, which is focused on longer horizon forecasting, we examine forecasting over horizons from one day to one month (the one-month horizon being where micro and macro analysis begin to overlap). Over our 3-year forecasting sample, we find that the micro-based model consistently out-performs both the random walk and the macro model. Micro-based forecasts account for almost 16 per cent of the sample variance in monthly spot rate changes. These results provide a level of empirical validation as yet unattained by other models. Our result that the micro-based model out-performs the macro model does not imply that macro fundamentals will never explain exchange rates. Quite the contrary, our findings are in fact consistent with the view that the principal driver of exchange rates is standard macro fundamentals. In Evans and Lyons (2004b)we report firm evidence that the non-public information that we exploit here for forecasting exchange rates is also useful for forecasting macro fundamentals themselves.

Martin D. D. Evans

Georgetown University

Department of Economics

Washington DC 20057

and NBER

evansm1@georgetown.edu

Richard K. Lyons

U.C. Berkeley

Haas School of Business

Berkeley, CA 94720-1900

and NBER

lyons@haas.berkeley.edu 


\section{Introduction}

This paper compares the true, ex-ante forecasting performance of a micro-based model against both a standard macro model and a random walk. In contrast to existing literature, which is focused on longer horizon forecasting, we examine forecasting over horizons from one day to one month (the one-month horizon being where micro and macro analysis begin to overlap). Over our 3-year forecasting sample, we find that the micro-based model consistently out-performs both the random walk and the macro model. Micro-based forecasts account for almost 16 per cent of the sample variance in monthly spot rate changes. These results provide a level of empirical validation as yet unattained by other models.

The forecasting experiment proposed by Richard Meese and Kenneth Rogoff (1983) remains a benchmark against which exchange rate models are judged. Their result that structural macro models cannot out-perform a naive random-walk has proven robust over the decades. Yet, the Meese-Rogoff paper was never about forecasting in the true sense, i.e., using time- $t$ information to forecast exchange rates at $t+1$. By using concurrent, realized values of the forcing variables, their regressions were more about concurrent explanation than about ex-ante forecasting. Their only "forecasting" element is in their reliance on ex-ante data to estimate equation parameters, which appropriately penalized models whose estimated parameters were unstable.

Charles Engel and Kenneth West (2004a,b) provide a valuable perspective on the forecastability of exchange rates. One explanation for the random walk nature of exchange rates is that there exists some unobserved fundamental that itself follows a random walk (unobserved being important because fundamentals proposed in most macro models do not follow random walks). They offer a different explanation, one rooted in the asset approach to exchange rates and the present value relation that follows from it. Specifically, they show that 
if fundamentals are $\mathrm{I}(1)$, but not necessarily random walks, then as the discount factor in the present value relation approaches one, the exchange rate will follow a process arbitrarily close to a random walk. Intuitively, given that an I(1) process can be split into random-walk and stationary components, a discount factor near one means that most all of the weight is placed on fundamentals far into the future, expectations of which are dominated by the random-walk component.

This paper takes the analysis of Engel and West as an important reorientation of thinking and brings it to the natural next step. Specifically, if there is little room for forecasting based on stationary components of fundamentals, then one needs to focus on where all the action is, namely, exchange rate dynamics that come from expectational surprises. Though the surprise part is, by definition, orthogonal to public information, our micro-based model shows that there should exist types of "non-public" information that are useful for forecasting this part, and where to look for these types of information. ${ }^{1}$ We then locate data on these types of non-public information and test whether they have true, ex-ante forecasting power. We should emphasize that our tests are qualitatively stronger than those of Meese and Rogoff: the hurdle for finding that a model outperforms a random walk is even higher.

Importantly, our result that the micro-based model out-performs the macro model does not imply that macro fundamentals will never explain exchange rates. Quite the contrary, our findings are in fact consistent with the view that the principal driver of exchange rates is standard macro fundamentals. As a conceptual matter, we show this by providing a structural interpretation - using a fundamentals-based model - that our findings are consistent with fundamentals being the driver. As an empirical matter, there is firm evidence that

\footnotetext{
${ }^{1}$ We refrain from using the word "news" because it has too strong an association with macro information that is public, which, even in the most careful of event studies, explains less that 5 percent of exchange rate variation in total (see Andersen et al. 2003).
} 
the non-public information that we exploit here for forecasting exchange rates is also useful for forecasting macro fundamentals themselves, so this information is not orthogonal to the evolving real economy (see Martin Evans and Richard Lyons 2004b).

The remainder of the paper has four sections. Section I provides perspective on why forecasting exchange rates is so difficult. Section II presents two forecasting models from the macro tradition and one forecasting model from the more recent, micro-based tradition. Section III describes our data, reviews some estimation issues, and presents our empirical results. Section IV presents concluding remarks.

\section{Forecasting Exchange Rates}

Why are future changes in exchange rates so hard to forecast? To address this question, we start with the present value expression for the spot rate:

$$
s_{t}=(1-b) \sum_{i=0}^{\infty} b^{i} E_{t} f_{t+i},
$$

where $s_{t}$ is the log nominal exchange rate (\$/other), and $f_{t}$ is current macro fundamentals. This equation nests a large class of macro exchange rate models: The precise definition of fundamentals and the specific form of the parameter $b$ depend on the macro model in question. To study the forecasting implications of (1), we iterate forward to get,

$$
s_{t}=E_{t} f_{t}+\frac{b}{1-b} E_{t} \Delta s_{t+1},
$$


and then rearrange to give:

$$
\Delta s_{t+1}=\frac{1-b}{b}\left(s_{t}-E_{t} f_{t}\right)+\varepsilon_{t+1}
$$

where:

$$
\varepsilon_{t+1} \equiv(1-b) \sum_{i=0}^{\infty} b^{i}\left(E_{t+1}-E_{t}\right) f_{t+i+1}
$$

Equations (2) and (3) allow us to analyze how the behavior of fundamentals affects the forecastability of exchange rates. To this end, let us start with the simple case of fundamentals $f_{t}$ following a random walk. Equation (1) implies that $s_{t}=E_{t} f_{t}$ in this case, so according to (2) the spot rate must also follow a random walk. The point here is that unforcastability in spot rates does not in itself imply that spot rates are disconnected from fundamentals. Put differently, forecasting success is not necessary for validating macro models.

Many macro models identify elements of $f_{t}$ as $I(1)$, but not random walks. In this case there should be some forecastability. However, Engel and West (2004a) point out that forecasting will still be hard because the value of $b$ implied by macro models is close to unity. We can illustrate their point with a simple example. Suppose that changes in fundamentals follow the auto-regressive process

$$
\Delta f_{t}=\phi \Delta f_{t-1}+u_{t}
$$

with $1>\phi>0$, and also that $E_{t} f_{t}=f_{t}$ (i.e. there is complete information about the current state of fundamentals). Then (1) implies that $s_{t}-f_{t}$ follows an $\mathrm{AR}(1)$ process

$$
s_{t}-f_{t}=\phi\left(s_{t-1}-f_{t-1}\right)+\frac{b \phi}{1-\beta \phi} u_{t}
$$


and $\varepsilon_{t+1}=\frac{1}{1-b \phi} u_{t+1}$. These results imply a theoretical $R^{2}$ from equation (2) of

$$
\frac{(1-b)^{2} \phi^{2}}{(1-b)^{2} \phi^{2}+\left(1-\phi^{2}\right)} \text {. }
$$

Simple calculations show that the implied values for $R^{2}$ are below 0.01 when $b$ is greater than 0.95 and $\phi$ is less than 0.8 . There is very little forecastability in $\Delta s_{t+1}$ when $b$ is close to unity unless the changes in fundamentals are very strongly autocorrelated.

In reality, forecasting exchange rates will be even harder than this example implies because we do not have data on the real-time estimates of fundamentals, $E_{t} f_{t}$. Even when we have the "correct" definition of fundamentals and so can construct a value for $f_{t}$, this ex-post value may differ significantly from the real time estimate (see, for example, Evans 2004). Under these circumstances, estimating $\Delta s_{t+1}=\frac{1-b}{b}\left(s_{t}-f_{t}\right)+\hat{\varepsilon}_{t+1}$ will produce poorer results than our example suggests because bias from measurement error pushes the coefficient on $s_{t}-f_{t}$ towards zero.

Engel and West's analysis and our simple example suggest that forecasting future spot rate changes with the fundamentals found in macro models is indeed a challenge. This does not mean that forecasting spot rates is all but impossible. On the contrary, it suggests that we reorientate our thinking towards a new class of models. Specifically, if there is little room for forecasting based on $s_{t}-f_{t}$, because $b$ is close to unity and changes in fundamentals are not very predictable, then we need to focus on where all the action is, namely, exchange rate dynamics that come from expectational surprises (i.e., $\varepsilon_{t+1}$ in equation 3 ). This is precisely the focus of more recent micro-based models. Macro models are agnostic about the process by which information is incorporated into new forecasts (since all relevant information is assumed public, and the mapping to price is direct and immediate). Micro-based models focus, by contrast, on the process through which dispersed information becomes known to the 
agents who set prices - the marketmakers - and is thereby incorporated in their expectations. Only after being incorporated in marketmaker expectations does it affect the prices that marketmakers set.

\section{Forecasting Models}

In this section we present the two models we shall use in our forecast comparisons. The first is representative of a wide class of macro-economic models. The second is a micro-based model - a stylized version of the model described in Evans and Lyons (2004a). Both models produce a present value representation for the log spot rate similar to equation (1).

\section{II.I Macro Models}

The macro model we examine has two central features: (i) the discount factor $b$ is very close to unity and (ii) information about future fundamentals arrives simultaneously to all agents, who in turn revise their forecasts for fundamentals in unison. Engel and West (2004b) note that feature (i) is a property of two of the most important models used in macro: the money-income model and Taylor-rule model. In the former, fundamentals are given by

$$
f_{t}=m_{t}-m_{t}^{*}-\gamma\left(y_{t}-y_{t}^{*}\right)+q_{t}-\left(v_{t}-v_{t}^{*}+\alpha \rho_{t}\right)
$$

where $m_{t}$ is the log nominal money supply, $y_{t}$ is log nominal output, $q_{t}$ is the real exchange rate, and $\rho_{t}$ is the FX risk premium (a "**" denotes a foreign variable). This specification produces a discount factor $b$ that equals $\alpha /(1+\alpha)$, where $\alpha$ is the interest semi-elasticity of money demand in both countries. Engel and West note that estimates of $\alpha$ in the literature of between 20 and 60 imply that $b$ is very close to unity. When interest rates are set according 
to a Taylor-rule, fundamentals are

$$
f_{t} \equiv\left(p_{t}-p_{t}^{*}\right)-\frac{1}{\varphi_{0}}\left[\rho_{t}+\varphi_{1}\left(y_{t}^{g}-y_{t}^{* g}\right)+\varphi_{2}\left(\pi_{t}-\pi_{t}^{*}\right)+u_{t}-u_{t}^{*}\right]
$$

where $p_{t}$ is the log nominal price level, $y_{t}^{g}$ is the "output gap", and $\pi_{t}$ is inflation. In this case the discount factor $b$ equals $\left(1+\varphi_{0}\right)^{-1}$, where $\varphi_{0}$ is the coefficient on the spot rate in the Taylor interest-rate rule. Engel and West point out that the values for $\varphi_{0}$ implied by actual monetary policy rules are small so that the discount factor $b$ must again be close to one.

Given the arguments above, there is little point in trying to forecast $\Delta s_{t+1}$ using $s_{t}-f_{t}$. Instead we make use of another feature of both the money-income and Taylor-rule models, the UIP equation: $E_{t} \Delta s_{t+1}=i_{t}-i_{t}^{*}+\rho_{t}$. Notice that if either model is correct, $i_{t}-i_{t}^{*}+\rho_{t}=$ $\frac{1-b}{b}\left(s_{t}-E_{t} f_{t}\right)$. Combining this expression with equation (2) gives us the ex-post version of the UIP equation:

$$
\Delta s_{t+1}=i_{t}-i_{t}^{*}+\rho_{t}+\varepsilon_{t+1}
$$

It is important to stress that this equation is just as good a specification as (2) from a forecasting point of view. From a practical standpoint, both equations require an assumption about the risk premium. We consider macro forecasts based on two assumptions: $(\mathrm{A}) \rho_{t}=\rho$ and (B) $\rho_{t}=\varphi_{0}-\varphi\left(i_{t}-i_{t}^{*}\right)$. Both specifications are encompassed by the regression:

$$
\Delta s_{t+1}=a_{0}+a\left(i_{t}-i_{t}^{*}\right)+\varepsilon_{t+1}
$$

Under (A), we have UIP with a constant risk premium: $a_{0}=\rho$ and $a=1$, and under (B) deviations in UIP are perfectly correlated with the interest differential $a_{0}=\varphi_{0}$, and $a=$ 
$1-\varphi$. We shall refer to (6) as the UIP model when assumption (A) holds, and as the Fama model when assumption (B) holds.

\section{II.II A Micro-Based Model}

Exchange rate dynamics in the micro-based model also focus on the present value relation. However, in this context there is a rather subtle but significant difference: the relevant expectation is that of the marketmaker. Specifically,

$$
s_{t}=(1-b) \sum_{i=0}^{\infty} b^{i} E_{t}^{m} f_{t+i}
$$

Here the present value relation characterizes the form of the price-setting rule for marketmakers; $E_{t}^{m}$ denotes expectations conditioned on marketmakers' information at the start of period $t$. This is a crucial difference because micro-based models focus on the process by which marketmakers obtain information (micro-level learning, in contrast to the symmetricinformation learning in traditional macro models). In particular, rewriting (7) we have:

$$
\begin{aligned}
\Delta s_{t+1} & =\left(\frac{1-b}{b}\right)\left(s_{t}-E_{t}^{m} f_{t}\right)+\varepsilon_{t+1}^{m} \\
\varepsilon_{t+1}^{m} & \equiv(1-b) \sum_{i=0}^{\infty} b^{i}\left(E_{t+1}^{m}-E_{t}^{m}\right) f_{t+i+1}
\end{aligned}
$$

Thus, innovations in spot rates, $\varepsilon_{t+1}^{m}$, come from the present value of revisions in marketmaker forecasts of future fundamentals.

Micro-based models tell us that marketmakers obtain information about fundamentals from the flow of transactions, specifically order flow. ${ }^{2}$ The idea is simple: If the trades of

\footnotetext{
${ }^{2}$ Order flow is signed transaction flow. Trades are signed from the direction of the initiating counterparty.
} 
private agents convey information about future fundamentals that is not currently known to marketmakers, then marketmakers will learn from those trades. Note that for the trades of private agents to convey information, it is not necessary that private agents perceive themselves to have superior information: they could instead be trading for purely allocational motives (e.g., clearing export transactions or repatriating earnings from abroad), with the sum of these trades conveying information about the macro-economy that is not otherwise available at the time. When a large number of agents are trading for correlated reasons, the resulting transaction flow during period $t$ (after $s_{t}$ is set) will convey information to marketmakers that causes them to revise their fundamentals forecasts.

Let us now consider the implications of the micro model for forecasting. First, note that any variable correlated with the arrival of information to marketmakers will be correlated with the exchange rate innovation $\varepsilon_{t+1}^{m}$. Thus, transaction flows during period $t$ seem an obvious candidate. There is strong empirical support for this idea in the data (Evans and Lyons 2002a,b). But from a forecasting point of view, this does not get us far. Rather, we need to understand whether transactions flows generated before period $t$ could be correlated with the arrival of information between the start of periods $t$ and $t+1$. If every marketmaker observed all transactions flows contemporaneously, the exchange rate innovation $\varepsilon_{t+1}^{m}$ should only be correlated with the unanticipated portion of transactions flows during period $t$. As a result, $\varepsilon_{t+1}^{m}$ should not be correlated with transaction flows during periods $t-1$ and earlier.

In sum, the forecasting power of micro-based models rest to two critical features. First, transactions flows must contain information relevant for fundamentals. This feature arises in an environment where either (1) agents initiating trades in the FX market have information they believe they can take advantage of; or (2) agents are trading for allocative reasons and the aggregate of those trades correlates with the current state of the macroeconomy. The 
second feature concerns the delay between the time information first generates transaction flows and the time this fact is widely recognized by marketmakers. If there is no delay because marketmakers can observe aggregate order flow contemporaneously, then spot rates will be correlated contemporaneously with order flow (as in Evans and Lyons 2002a). The forecasting power of order flow arises precisely because it takes time for the implications of aggregate order flow to be recognized across all marketmakers and hence reflected in spot prices.

We can illustrate how information in order flow may be delayed with a simple example. Suppose marketmakers observe only part of the aggregate order flow in real time, and learn fully about aggregate order flow with a lag. In particular, let us assume that aggregate order flow during period $t, x_{t+1}$, follows an $\mathrm{AR}(1)$ process: $x_{t+1}=\lambda x_{t}+v_{t+1}$ where $v_{t+1}$ is a i.i.d. mean zero shock with variance $\sigma_{v}^{2}$. Further, assume that each marketmaker (indexed by $i$ ) observes a sample of aggregate order flow $x_{t+1}^{i}$ during period $t$ trading where $x_{t+1}^{i}=x_{t+1}+\xi_{t+1}^{i}$ and $\xi_{t+1}^{i}$ is a i.i.d. mean zero idiosyncratic shock with variance $\sigma_{\xi}^{2}$. We also assume that marketmakers learn the true aggregate order flow with a one-period delay. Thus, at the start of period $t$, marketmaker $i$ 's information $\Omega_{t}^{i}$ comprises the history of aggregate order flow $\left\{x_{t-1}, x_{t-2}, \ldots\right\}$ and individual order flow $\left\{x_{t}^{i}, x_{t-1}^{i}, x_{t-2}^{i}, \ldots\right\}$. In this environment, the unexpected order flow observed by marketmaker $i$ during period $t$ trading can be shown to be:

$$
\begin{aligned}
x_{t+1}^{i}-E_{t}^{i} x_{t+1}^{i} & =x_{t+1}-E_{t}^{i} x_{t+1}+\xi_{t+1}^{i}, \\
& =v_{t+1}+\lambda \psi v_{t}+\xi_{t+1}^{i},
\end{aligned}
$$


where $\psi \equiv \sigma_{\xi}^{2} /\left(\sigma_{v}^{2}+\sigma_{\xi}^{2}\right)$ and $E_{t}^{i}$ denotes expectations conditioned on $\Omega_{t}^{i}$. Thus, the order flow information received by marketmaker $i$ has an aggregate component $v_{t+1}$ that follows an $\mathrm{MA}(1)$ and an idiosyncratic component $\xi_{t+1}^{i}$. This means that the information received by individual marketmakers during period $t$ trading will be correlated with past innovations in aggregate order flow $v_{t}$.

Does this mean that the forecast revisions $\varepsilon_{t+1}^{m}$ can be correlated with $v_{t}$ ? Not necessarily. It depends on whether marketmakers would find it optimal to change their own price quotes in the face of idiosyncratic information. This is not the case in models of marketmaker behavior (Lyons 1997 and Evans and Lyons 2002a). In the Bayes-Nash equilibrium of these models, each marketmaker chooses to trade on idiosyncratic information (at the prices quoted by other marketmakers) rather than simply announcing it to others in the form of a price quote. Moreover, if differences between the quotes set by marketmakers in a given period make them vulnerable to being arbitraged, marketmakers have a further reason not to change their quotes in response to idiosyncratic information. In the context of our example, this Bayes-Nash logic means that marketmakers would not change their quote between $t$ and $t+1$ based on $x_{t+1}^{i}-E_{t}^{i} x_{t+1}^{i}$ because it contains idiosyncratic information $\xi_{t+1}^{i}$. Rather, they would wait until they have a more precise signal, one that is purged of $\xi_{t+1}^{i}$. This is achieved at the start of period $t+1$ when they learn $x_{t}$. When all marketmakers learn $x_{t}$, the value of $v_{t}$ becomes common knowledge.

We can put these ideas together in a stylized model of fundamentals and order flow:

$$
\begin{aligned}
\Delta f_{t} & =\phi \Delta f_{t}+u_{t}+\delta v_{t}, \\
x_{t} & =\lambda x_{t-1}+v_{t} .
\end{aligned}
$$


Equation (10a) extends our specification of the fundamentals process in equation (4). Innovations in fundamentals growth include a common-knowledge component $u_{t}$, and a component correlated with the innovation in aggregate order flow, $v_{t}$. We assume that $u_{t}$ is observed contemporaneously (i.e. at the start of period $t$ ), and that $v_{t}$ only becomes known to all marketmarkers with a one period delay. Under these assumptions, marketmakers do not know the concurrent state of the macroeconomy. Rather $E_{t}^{m} f_{t-1}=f_{t-1}$, and $E_{t}^{m} f_{t}=(\phi+1) f_{t-1}-\phi f_{t-2}+u_{t}$, so $f_{t}-E_{t}^{m} f_{t}=\delta v_{t}$. With these results we can rewrite equation (7) as

$$
s_{t}=E_{t}^{m} f_{t}+\frac{b \phi}{1-b \phi} E_{t}^{m} \Delta f_{t}=\frac{1}{1-b \phi} f_{t}-\frac{b \phi}{1-b \phi} f_{t-1}-\frac{\delta}{1-b \phi} v_{t},
$$

so the innovation in spot rates $\varepsilon_{t+1}^{m} \equiv s_{t+1}-E_{t}^{m} s_{t+1}$ is:

$$
\varepsilon_{t+1}^{m}=\frac{1}{1-b \phi}\left(f_{t+1}-E_{t}^{m} f_{t+1}\right)-\frac{b \phi}{1-b \phi}\left(f_{t}-E_{t}^{m} f_{t}\right)-\frac{\delta}{1-b \phi} v_{t+1}=\frac{1}{1-b \phi} u_{t+1}+\frac{[1+\phi(1-b)] \delta}{1-b \phi} v_{t} .
$$

Finally, substituting for $v_{t}$ and combining the result with (8a) gives the following forecasting equation:

$$
\Delta s_{t+1}=\frac{1-b}{b}\left(s_{t}-E_{t}^{m} f_{t}\right)+\frac{1}{1-b \phi} u_{t+1}+\frac{[1+\phi(1-b)] \delta}{1-b \phi}\left(x_{t}-\lambda x_{t-1}\right) .
$$

This equation shows that lagged order flows can have forecasting power for spot rates even when the discount factor is very close to unity: the coefficient on the last term has a limiting value of $\delta /(1-\phi)$ as $b \rightarrow 1$.

In the empirical analysis below we consider two forecasting equations based on (11). The first specification is given by

$$
\Delta s_{t+1}=a_{0}+a x_{t}^{\mathrm{AGG}}+e_{t+1},
$$


where $x_{t}^{\mathrm{AGG}}$ denotes aggregate order flow from six end-user segments. The second specification considers the forecasting power of disaggregated order flow:

$$
\Delta s_{t+1}=a_{0}+\sum_{j=1}^{6} a_{j} x_{j, t}^{\mathrm{DIS}}+e_{t+1}
$$

where $x_{j, t}^{\mathrm{DIS}}$ denotes the order flow from segment $j$. Notice that the transaction flows in both specifications are derived from trading that took place before the start of period $t .{ }^{3}$ Below we refer to the specificaions in (12) and (13) as the Micro I and Micro II models.

One further point deserves emphasis: the fact that aggregate order flow has forecasting power for spot rates is not a violation of market efficiency (at least not in the weak or semi-strong senses of efficiency). As researchers, we have access to aggregate order flow data that was not part of the information set available to all marketmakers at the time they were setting spot rates. Ex post, forecastability looks like a missed opportunity for profit (though not necessarily on a risk-adjusted basis). Ex ante, however, it is important to recognize that this order flow information is not available to everyone.

\section{Empirical Analysis}

\section{III.I Data}

Our empirical analysis utilizes a new data set that comprises end-user transaction flows, spot rates and euro deposit rates over six and half years. The transaction flow data is of a fundamentally different type and it covers a much longer time period than the data used

\footnotetext{
${ }^{3}$ Equations (12) and (13) do not include the interest differential to control for the first term on the right hand side of (11). Adding the interest differential has no effect on the forecasting performance of the micro-based models because, as we shall see, the differential has no forecasting power whatsoever.
} 
in earlier work (e.g., Evans and Lyons 2002a,b). The difference in type is our shift from inter-marketmaker order flow to end-user order flow. By end users we are referring to three main segments: non-financial corporations, investors (such as mutual funds and pension funds), and leveraged traders (such as hedge funds and proprietary traders). The data set also contains information on trading location. From this we construct order flows for six end-user segments: trades executed in the US and non-US markets for non-financial firms, investors, and leveraged traders. Though inter-marketmaker transactions account for about two-thirds of total volume in major currency markets, they are largely derivative of the underlying shifts in end-user demands. Our data on the end-user segments include all of Citibank's end-user trades in the largest spot market, the USD/EUR market, from January 1993 to June 1999. ${ }^{4}$ The data also include all of Citibank's end-user trades in the USD/EUR forward market over the same period. Citibank's end-user market share in these currencies is in the 10-15 percent range; no other bank has a larger market share in these currencies.

\section{III.II Forecast Comparisons}

We test for forecastability in two ways. First, we report mean-squared error (MSE) ratios to be comparable with earlier studies, notably Nelson Mark (1995) and Jon Faust et al. (2003). We also construct a new test statistic for comparing the forecasting performance of models relative to the random walk (RW). ${ }^{5}$ This statistic, the "projection" statistic, is both easy to compute and has a straightforward asymptotic distribution under the null that the exchange rate follows a random walk. It also provides an economically meaningful measure

\footnotetext{
${ }^{4}$ Before January 1999, data for the euro are synthesized from data in the underlying markets against the dollar, using weights of the underlying currencies in the euro.

${ }^{5}$ We construct this test because conventional statistics based on MSE's as computed by Christiano (1989), Mark (1995) and others have a complicated (non-normal) asymptotic distribution when the models are nested, as they are here (Clark and McCraken 2001).
} 
of forecasting performance. All of our results are based on recursive estimates of the non-RW model using a growing number of observations.

We compute the projection statistic as follows: Let $\Delta^{h} s_{t+h \mid t}$ denote the forecast of $\Delta^{h} s_{t+h} \equiv s_{t+h}-s_{t}$ computed from a model estimated with data available on day $t$. First we construct the recursive $h$-period out-of-sample forecasts for the non-RW model over the forecasting period starting on day $S$ and ending on day $T-h:\left(i . e ., \widehat{\Delta s_{t+h \mid t}}\right.$ for $\left.S<t \leq T-h\right)$. Next we regress the forecasts on the realized values for $\Delta s_{t+h}$ :

$$
\widehat{\Delta s_{t+h \mid t}}=\beta_{0}+\beta \Delta s_{t+h}+w_{t+h}
$$

Under the null hypothesis that $s_{t}$ follows a random walk, there is no forecast change in the spot rate so $\Delta s_{t+h \mid t}=0$. Under these circumstances, the out-of-sample forecasts based on the alternative model estimates, $\widehat{\Delta s_{t+h \mid t}}$, only differ from zero due to the presence of sampling error. Furthermore, since these forecasts are calculated using data up to day $t$, the error cannot be correlated with $\Delta s_{t+h}$ under the null. These observations imply that we can compare forecasting performance of a model against the random walk benchmark, simply by testing for the significance of the $\beta$ coefficient. If the alternate model does no better than the RW, we should find estimates of $\beta$ insignificantly different from zero. If the alternate model does have forecasting power, then the estimates of $\beta$ should be positive and significant. The only factor complicating inference concerns the possible presence of serial correlation in $w_{t+h}$. We compute an estimate of the variance of $\beta$ using the Newey-West (1987) estimator. ${ }^{6}$

\footnotetext{
${ }^{6}$ Consistency of the standard error on $\beta$ requires that the truncation lag used in the covariance estimator increases with the sample size. In practice we set the truncation lag to $h-1$.
} 


\section{III.III Results}

Our results are reported in Table 1. The table presents the forecasting power of four different models: the UIP and Fama models based on equation (6), the Micro I model specified in (12), and the Micro II model specified in (13). These models are compared across 5 different forecasting horizons $h: 1,5,10,15$, and 20 trading days, using 6/3/1996 as the start of the forecasting period. ${ }^{7}$ Note that 20 trading days is 4 trading weeks, i.e., roughly one calendar month. In the UIP and Fama models we use euro deposit rates with maturities that match the forecast horizon. In the micro-based models order flows are derived from transactions over the $h$ trading days starting on day $t-h$.

Table 1 shows that the forecasting performance of the macro models is uniformly poor, in keeping with results from Meese and Rogoff (1983) and the voluminous literature that followed their work (for a recent update, see Yin-Wong Cheung et al. forthcoming). In contrast, the forecasting performance of the micro models is significantly better, particularly as the forecasting horizon is extended. According to the projection statistic, the forecasting ability of the Micro I model is significantly better than the RW model at the one percent level at horizons of 10 days or longer. The results from the Micro II model are if anything even stronger. The projection statistics indicate that disaggregated order flow has statistically significant forecasting power for spot rate changes at all horizons. This finding is robust to our forecasting method. We find similar results when forecasts are based on rolling estimates of the Micro II model using a fixed number of observations.

The estimates of $\beta$ also provides us with a more economically meaningful measure of the

\footnotetext{
${ }^{7}$ The number of out-of-sample forecasts used to compute the MSE and projection statistics for $h=$ $\{1,5,10,15,20\}$ are $797,793,788,783$, and 778 , respectively. Since there are at least 38 non-overlapping observations $(778 / 20>38)$ in the forecasting period, our results should be largely immune to the well-known small-sample problems that plague inference in long-horizon forecast comparisons conducted over standard data spans.
} 
Table 1: Forecast Comparisons

\begin{tabular}{|c|c|c|c|c|c|}
\hline & \multicolumn{5}{|c|}{ Horizon $h$ (trading days) } \\
\hline & 1 & 5 & 10 & 15 & 20 \\
\hline \multicolumn{6}{|l|}{ UIP } \\
\hline MSE & 1.001 & 1.006 & 1.012 & 1.016 & 1.021 \\
\hline p-value & $(1.000)$ & $(1.000)$ & $(1.000)$ & $(1.000)$ & $(1.000)$ \\
\hline$\beta$ & 0.000 & 0.000 & 0.000 & 0.000 & 0.000 \\
\hline $\mathrm{p}$-value & $(0.058)$ & $(0.597)$ & $(0.542)$ & $(0.488)$ & $(0.414)$ \\
\hline \multicolumn{6}{|l|}{ Fama } \\
\hline MSE Ratio & 1.005 & 1.011 & 1.022 & 1.035 & 1.054 \\
\hline p-value & $(1.000)$ & $(1.000)$ & $(1.000)$ & $(1.000)$ & $(1.000)$ \\
\hline$\beta$ & 0.000 & 0.003 & 0.002 & 0.003 & 0.010 \\
\hline $\mathrm{p}$-value & $(0.533)$ & $(0.332)$ & $(0.457)$ & $(0.452)$ & $(0.359)$ \\
\hline \multicolumn{6}{|l|}{ Micro I } \\
\hline MSE Ratio & 1.026 & 1.015 & 1.001 & 0.946 & 0.896 \\
\hline p-value & $(1.000)$ & $(1.000)$ & $(1.000)$ & $(0.357)$ & $(0.106)$ \\
\hline$\beta$ & 0.002 & 0.024 & 0.092 & 0.133 & 0.129 \\
\hline $\mathrm{p}$-value & $(0.398)$ & $(0.118)$ & $(0.000)$ & $(0.000)$ & $(0.000)$ \\
\hline \multicolumn{6}{|l|}{ Micro II } \\
\hline MSE Ratio & 0.961 & 0.876 & 0.848 & 0.810 & 0.806 \\
\hline p-value & $(0.124)$ & $(0.024)$ & $(0.091)$ & $(0.045)$ & $(0.055)$ \\
\hline$\beta$ & 0.027 & 0.057 & 0.102 & 0.122 & 0.157 \\
\hline $\mathrm{p}$-value & $(0.005)$ & $(0.018)$ & $(0.005)$ & $(0.007)$ & $(0.002)$ \\
\hline
\end{tabular}

Notes: MSE ratio is the ratio of mean squared forecast errors for the non-RW model to the RW model. The p-value from a one-sided test for the RW null is reported in parenthesis under the MSE ratios. These p-values are computed as in Mark (1995) with the Andrews $\mathrm{AR}(1)$ rule for the truncation lag The p-values below the estimates of $\beta$ are for the null $\beta=0$ and are computed from the asymptotic distribution of the OLS estimates using Newey-West estimator with $h-1$ lags. 
forecasting performance. By definition, the $h$ - period change in the spot rate comprises a forecastable and unforecastable component: $\Delta s_{t+h} \equiv \widehat{\Delta s_{t+h \mid t}}+\widehat{\varepsilon_{t+h \mid h}}$. Multiplying both sides of this identity by $\Delta s_{t+h}$, and taking expectations gives us a variance decomposition for spot rate changes:

$$
\operatorname{Var}\left(\Delta s_{t+h}\right)=\operatorname{Cov}\left(\Delta s_{t+h}, \widehat{\Delta s_{t+h \mid t}}\right)+\operatorname{Cov}\left(\widehat{\varepsilon_{t+h \mid h}}, \Delta s_{t+h}\right)
$$

Since the projection coefficient $\beta$ is simply the ratio of the covariance between $\Delta s_{t+h}$ and

$\widehat{\Delta s_{t+h \mid t}}$ to the variance of $\Delta s_{t+h}$, the values for $\beta$ reported in Table 1 estimate the contribution of the model forecasts to the variance of spot rate changes over the forecasting period. As the table shows, forecast based on either micro-based model account for a greater fraction of the variance in spot rates as the forecasting horizon rises. Forecasts from the Micro II model account for almost 16 per cent of the sample variance in monthly spot rate changes. By this metric, the forecasting power of disaggregated order flows is truly significant from an economic perspective.

\section{Conclusions}

Within the public information paradigm, which is standard in exchange rate economics, the scope for successful exchange rate forecasting is narrow indeed: forecasts based on exante expected changes perform poorly (Meese and Rogoff 1983, Engel and West 2004b) and forecasting surprises is, by definition, impossible from pubic information. Relaxing complete information offers, at least at the conceptual level, a way to proceed. We provide a model showing why there may exist types of non-public information that should be useful for forecasting exchange rate surprises (i.e., price changes that cannot be explained based on 
measures of public information). Because our model provides a structural interpretation that is fundamentals based, this shows that surprises being forecastable is consistent with the bedrock idea that exchange rates are driven by macro fundamentals.

The proof is, of course, in the empirical pudding. When we compare the true, ex-ante forecasting performance of a micro-based model against both a standard macro model and a random walk, we find that the micro-based model consistently out-performs both: microbased forecasts account for roughly 16 percent of the variance in monthly spot rate changes. Because our analysis is not based on concurrent, realized values of the forcing variables - as was that of Meese and Rogoff (1983) - the results provide a level of empirical validation as yet unattained by other models. Put differently, our tests are qualitatively stronger than those of Meese and Rogoff in the sense that the hurdle for finding that our micro-based model outperforms a random walk is even higher here. Moreover, as Evans and Lyons (2004b) shows, there is firm evidence that the non-public information that we exploit here for forecasting exchange rates is also useful for forecasting macro fundamentals themselves.

Future work along micro-based modeling lines might consider the following related issues. First, is the forecasting power here coming from the real economy (i.e., the non-financial firms)? If so, this would provide a still closer link to the relevance of supply-side factors in exchange rate determination (in the spirit of new open economy modeling). Second, if the dispersed information framework is the right one, what are the implications for deep issues such as market incompleteness, risk sharing, and goods-market pricing? Third, to what degree is the information being revealed in order flow actually macroeconomic information? Might it instead be due, at least in part, to variations in equilibrium expected returns? And if the latter, what are the links to existing models of risk-premium determination? 


\section{References}

Andersen, Torben; Bollerslev, Tim; Diebold, Francis and Vega, Clara. "Micro Effects of Macro Aannouncements: Real-time Price Discovery in Foreign Exchange." American Economic Review, 2003, 93, pp. 38-62.

Cheung, Yin-Wong; Chinn Menzie and Pascual, Antonio. "Empirical Exchange Rate Models of the Nineties: Are any Fit to Survive?" Journal of International Money and Finance, forthcoming.

Clark, Todd and McCraken, Michael. "Evaluating long-horizon forecasts." Federal Reserve Bank of Kansas City working paper No. 01-14, 2001.

Christiano, Lawrence. "P*: Not the Inflation Forecaster's Holy Grail," Federal Reserve Bank of Minneapolis Quarterly Review, 1989, 13 pp. 3-18.

Engel, Charles and West, Kenneth. "Exchange Rates and Fundamentals." National Bureau of Economic Research (Cambridge MA) Working Paper 10723, August 2004a, and Journal of Political Economy, forthcoming.

. "Accounting for Exchange Rate Variability in Present Value Models when the

Discount Factor is Near One." American Economic Review, May 2004b (Papers and Proceedings) 94, pp. 118-125.

Evans, Martin. "Where Are We Now? Real-Time Estimates of the Macro Economy." Mimeo, Georgetown University, 2004.

Evans, Martin and Lyons, Richard. "Order flow and Exchange Rate Dynamics." Journal of Political Economy, 2002a, 110, pp. 170-180.

-. "Informational Integration and FX Trading." Journal of International Money and Finance, 2002b, 21, pp. 807-831. 
_. "A New Micro Model of Exchange Rate Dynamics." National Bureau of Economic Research (Cambridge MA) Working Paper 10379, March 2004a. ."Exchange Rate Fundamentals and Order Flow." Mimeo, Georgetown University, 2004b.

Faust, Jon; Rogers, John and. Wright, Johnathan. "Exchange Rate Forecasting: The Eerrors we've Really Made." Journal of International Economics, 2003, 60, pp. 35-59.

Lyons, Richard. "A Simultaneous Trade Model of the Foreign Exchange Hot Potato." Journal of International Economics, 1997, 42 pp. 275-298.

Mark, Nelson. "Exchange Rates and Fundamentals: Evidence on Long-horizon Predictability." American Economic Review, 1997, 85 pp. 201-218.

Meese, Richard, and Rogoff Kenneth. "Empirical Exchange Rate Models of the Seventies." Journal of International Economics, 1983, 14 pp. 3-24.

Newey, Whitney. and West, Kenneth. "A Simple, Positive Semidefinite, Heteroskedasticity and Autocorrelation Consistent Covariance Matrix," Econometrica 1987, 55 pp. 703708. 